\title{
The Genetics of Childhood Disease and Development
}

The genomic revolution has changed our world and has affected and expanded the studies of childhood disease and human development. Accordingly, Pediatric Research is introducing a series of review articles to emphasize the importance of genomics in the study of the diseases and disorders of childhood. This series includes studies of unique applications of genomics in the control of respiration, cognition, developmental biology, and the understanding of the mechanisms of bacterial diversity.

The first article in our new genetics series, published in this issue, is "Genomics and Pediatric Research." In this article, Boright, Kere, and Scherer describe the excitement associated with and caused by the genomics revolution and the potential of applying new technology to the study of disease and the development of diagnostic tests and treatment. The authors suggest that we must be prepared to question some of the dogma of pediatrics, such as "one gene, one protein, one disease." Also we must recognize that the use of genomic-based technology means that much research will not be hypothesis-driven and experimental but rather observational and analytical. They consider in their discussion not only monogenic diseases but also multifactor etiologies involving several genes or the interaction of genes and environment. It is clear that genomics knowledge and technology will influence every field of pediatric research and they endeavor to remove the "hype" and emphasize the current stage of our knowledge.

Pediatric Research has long been a forum for publication of current studies of childhood disease and human development. It is not surprising, therefore, that the number of genomerelated articles published in Pediatric Research continues to increase. Boright, Kere, and Scherer point out that, in the past year, 23\% of the articles published in Pediatric Research used genomic information or technologies.

Genomics has already made major contributions to the diagnosis, understanding, treatment, and prevention of childhood disease. It will undoubtedly be an increasing component of the types of experimental and observational studies published in Pediatric Research. 